\section{What is theoretical biology?}

Lee A. Segel

Theoretical Biology and Complexity: Three Essays on the Natural Philosophy of Complex Systems. Edited by Robert Rosen. Academic: 1985. Pp.210. \$57, £48.

IN HIS preface, Robert Rosen states that the three scientists whose essays make up this book

each began with a conviction that contemporary physics already contained the necessary universals with which to cope with the phenomena of life and that therefore only a clever rearrangement and redeployment of these universals would suffice to bring them to bear effectively on biology. .... we each came separately, and with great reluctance, to admit the possibility that this conviction might not be true and hence that a true theory of the organism required new physics and new epistemology. And again separately, we realized that the measurement process, which lies at the very heart of every mode of system description, provides perhaps the only safe and fundamental point of departure for building a comprehensive theory, not only of organisms, but of natural systems in general.

I believe that most workers in theoretical biology are indifferent to, or disagree with, the main points just cited. But as I have no brief to speak for others, let me give my own views.

It is inappropriate to try to build comprehensive theories at this early stage in the modern study of biology. Rather, it is better to tackle particular phenomena and to try to generalize from the results. Although there is probably need to redress a present-day over-confidence in the comprehensiveness of conclusions that can be reached by molecular biology, the remedy is not to concentrate upon epistemology.

Certainly, an eventual aim should be to understand "the organism"; that is, answer the question "What is life?". But there are other, equally important and more approachable questions to be asked, such as "How does a baby develop from a fertilized egg?" or "How does the brain process, store and retrieve information?". Of course, new physics is needed for this enterprise - for example, even recent extensions of thermodynamics apply only to situations that are close to equilibrium. The major province of present-day theoretical biologists is to extend old concepts and introduce new ones in phenomenological fashion, at appropriately selected levels in the hierarchy of biological organization.

To my mind, saying that the measurement process is central to biology is to relapse into the sin of adopting too uncritically the ideas of physics. An example of

a more successful point of departure is the stress on computation in the study of vision by $\mathrm{D}$. Marr and his associates.

The authors are of course entitled to have their say. In an extension of classical formulations of mechanics and thermodynamics, I.W. Richardson discusses the interaction between particles and the consequent observable manifestations thereof. (There is no explicit mention of biology in his essay.) A.H. Louie tries to deal with problems of measurement, interaction and representation using category theory, a relatively recent branch of abstract algebra. (More than one hundred special symbols are listed at the beginning of the essay, signalling the fact that it will be comprehensible to few apart from mathematicians.) Here, too, there is little specific contact with biology.

Rosen's article does contain quite a bit about biology and is relatively accessible. But for two reasons I doubt the need for his urgings to go beyond the evolving state description that has been so successful in particle physics - such descriptions have in fact proved valuable in large areas of biology (for example in biochemical

\section{On the track of an epidemic}

\section{Richard S. Tedder}

Understanding AIDS: A Comprehensive Guide. Edited by Victor Gong. Rutgers University Press/Cambridge University Press:1985. Pp.240. Hbk \$20, £17.50; $p b k \$ 9.95$.

AIDS: The Acquired Immune Deficiency Syndrome. By Victor G. Daniels. MTP Press:1985. Pp.155. £9.95, \$16.25.

AIDS: Etiology, Diagnosis, Treatment and Prevention. By Vincent DeVita, Jr., Samuel Hellman and Steven Rosenberg. Lippincott/Harper \& Row:1985. Pp.352. $\$ 38, £ 41.95$.

To CALL Acquired Immune Deficiency Syndrome the infectious disease of the 1980 s may unhappily be wishful thinking - although this decade has seen the emergence of AIDS, it is most unlikely to see its departure. Initially thought to be limited to homosexual men, there is no doubt that the causative virus, HTLV III/LAV, is becoming widely established in the human population. It is especially unfortunate that the homosexual man bears the brunt of infection, since this has engendered both a moralistic and a narrow view of the disease. Moralistic, in the sense that people who do not have homosexual lovers can underline their disapproval by invoking Divine Retribution; narrow, in that a belief that the virus is confined to gay men encourages theories that exploit the law of mass action), and alternative formalisms have frequently been employed (for example graph theory in ecology).

The authors' point of view is best summed up by Dr Louie, who states that "I have always been more interested in 'conceptual' biomathematics than in looking at specific models of a biological process. In other words, my interest is oriented more toward the logic of mathematical biology". Those who share this attitude and who have a good mathematical background will find food for thought in this book. Those who are more pragmatically inclined should peruse the Journal of Theoretical Biology, Biophysical Journal, the "Models and Hypotheses" section of Differentiation or some of the more than 60 volumes in Springer-Verlag's series Lecture Notes in Biomathematics. Included in such publications, albeit embedded in inescapable chaff, are many specific kernels of understanding that theorists have brought to biology.

Lee A. Segel is a Professor in the Department of Applied Mathematics, Weizmann Institute of Science, Rehovot 76100, Israel.

complacency about public health.

It is the speed with which HTLV III/ LAV has established itself as an endemic infection within the human race that should be the main cause for concern, and not merely the increasing numbers of patients with AIDS. Taken alone, the figure for cases of AIDS is a gross underestimation of the prevalence of infection and the true size of the problem for the future. Early in any epidemic the initial cases of disease will be those with a short incubation and will underrepresent the clinical attack rate. There is now clear evidence that the virus may be transmitted by heterosexual intercourse and is not only passed on by homosexual activity. This is particularly true of the African epidemic which is itself of recent origin; moreover, women may transmit the disease to the next generation, either in utero or possibly through breast milk. The human race is facing a microbial threat which, rightly or wrongly, must call into question current attitudes to all sexual practices. It is surely not sufficiently comforting to remark that AIDS is essentially a disease of certain limited segments of the population when we know that infected persons may be both infectious and themselves subject to illness of diverse forms for an entire lifetime.

Set against this background, any book called Understanding AIDS: A Comprehensive Guide is in for a certain amount of scepticism. Where knowledge is accruing so fast, any book on the subject will be out of date as soon as it is published. However in this instance it may never have been "in date". Although the 